\title{
Bacillus Calmette-Guérin therapy-associated granulomatous prostatitis mimicking prostate cancer on MRI: A case report and literature review
}

\author{
NICOLAIE SUDITU ${ }^{1}$ and DRAGOS NEGRU ${ }^{2}$ \\ ${ }^{1}$ Department of Urology, Dr. C.I. Parhon Hospital, 700503 Iasi; \\ ${ }^{2}$ Department of Radiology, St. Spiridon Hospital, Gr. T. Popa University of Medicine, 700111 Iasi, Romania
}

Received July 18, 2014; Accepted September 12, 2014

DOI: $10.3892 / \operatorname{mco} .2014 .422$

\begin{abstract}
Granulomatous prostatitis following bacillus Calmette-Guérin (BCG) immunotherapy is a well-known pathological entity, developing following initiation of $\mathrm{BCG}$ therapy as a prophylactic measure against the recurrence of non-muscle invasive bladder cancer. In addition, there are certain imaging similarities between granulomatous prostatitis and prostate cancer, including hypoechoic area on transrectal ultrasonography and low T2 signal intensity in some prostate areas on magnetic resonance imaging (MRI). This is the report of a case exhibiting a perfect imaging cross-match between granulomatous prostatitis and potential prostate cancer on repeated MRI exams, adding two supplementary aspects to the already known similarities, namely progressive restricted diffusion and increased contrast enhancement, which are specific to prostate cancer.
\end{abstract}

\section{Introduction}

Magnetic resonance imaging (MRI) is an elective imaging investigation method used for the detection, staging and follow-up of prostate cancer patients. The specific MRI imaging characteristics of prostate cancer, i.e., low T2 signal intensity, restricted diffusion area, low apparent diffusion coefficient and contrast enhancement, suggest the diagnosis, enable TNM staging and may guide prostate biopsy. Therefore, we consider that, in patients with such MRI lesions, histopathological examination is required to confirm the diagnosis of malignancy, although prostate-specific antigen (PSA) levels and digital rectal examination (DRE) findings may be normal. The aim of this study was to demonstrate a high similitude

Correspondence to: Dr Nicolaie Suditu, Department of Urology, Dr. C.I. Parhon Hospital, 50 Carol I Blvd, 700503 Iasi, Romania E-mail: nicolaiesuditu@yahoo.com

Key words: bacillus Calmette-Guérin immunotherapy, granulomatous prostatitis, prostate cancer, magnetic resonance imaging, prostate biopsy between prostate cancer and an inflammatory disease, such as granulomatous prostatitis, on MRI examination.

\section{Case report}

A 64-year-old patient with a medical history of heavy smoking (43 pack-years index) and atrial fibrillation-flutter was diagnosed with a small $(3 \mathrm{~cm})$, solitary, non-muscle invasive bladder tumor. The bladder tumor was diagnosed as low-grade (G1) urothelial carcinoma, without invasion of the chorion (Ta). It is important to mention that, during transurethral resection of the blader (TURB), the patient developed a major cardiac arrhythmia, which ultimately required cardiac electroconversion. Despite the fact that the patient had a low risk of disease recurrence and progression (1), we recommended bacillus Calmette-Guérin (BCG) immunotherapy (Lamm protocol) (2), due to the smoking history and the high risk of anaesthesia due to the cardiac pathology. Following BCG induction treatment (6 weekly instillations), at 3 months after TURB the first control cystoscopy would have been recommended. However, as the patient exhibited a low level of pain tolerance, which would require a form of general anaesthesia, in order to avoid a possible recurrence of the cardiac arrhythmia, we decided to perform a pelvic MRI scan instead of a flexible cystoscopy.

The MRI revealed normal bladder mucosa but also revealed a $19 \times 27 \times 21 \mathrm{~mm}$ area in the peripheral region of the right prostatic lobe, exhibiting certain malignant characteristics, namely low T2 signal intensity, restricted diffusion (Fig. 1A) and contrast enhancement (Fig. 1B), compared to the rest of the prostate gland. The prostate had only been evaluated by digital rectal examination (DRE), abdominal ultrasonography and computed tomography (CT), without any findings suggestive of pathological lesions. PSA measurement had been postponed due to all the urethral manoeuvering. Therefore, 3 months after TURB and 1 month after BCG immunotherapy and the first MRI examination, the PSA level was borderline $(3.9 \mathrm{ng} / \mathrm{ml}$; normal range, $0-4 \mathrm{ng} / \mathrm{ml}$ ), with a prostate volume of $40.14 \mathrm{~cm}^{3}$. Therefore, we decided to continue with the clinical, biological and imaging monitoring of the bladder and prostatic lesions. Six weeks after the first stage of local immunotherapy induction, the patient underwent the second stage of BCG induction 


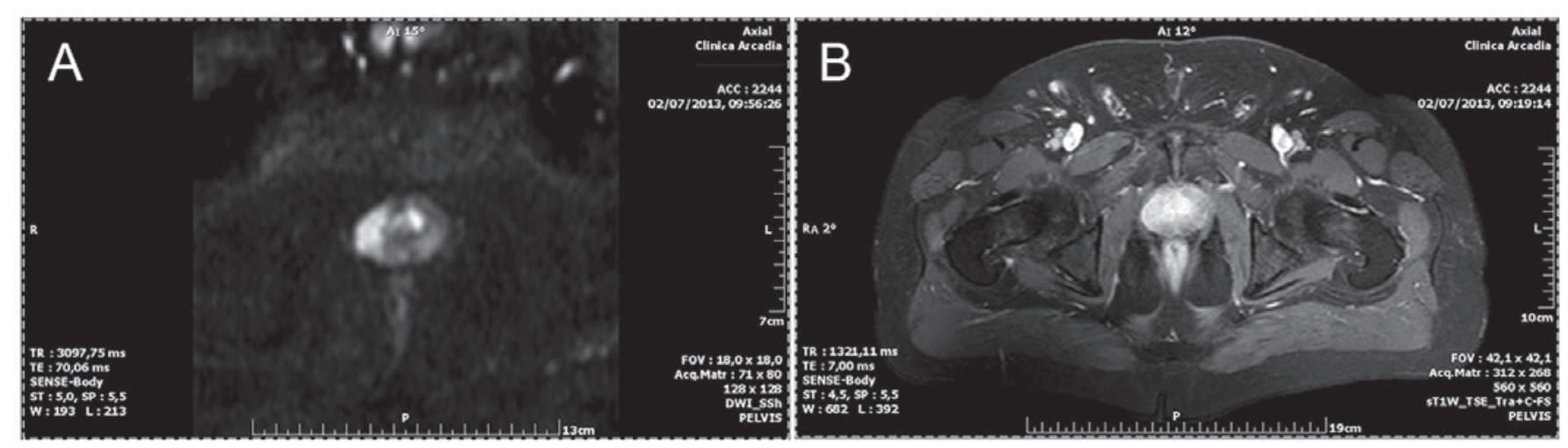

Figure 1. (A) Restricted diffusion area in the peripheral right prostate lobe. (B) Contrast enhancement area in the right prostate lobe.

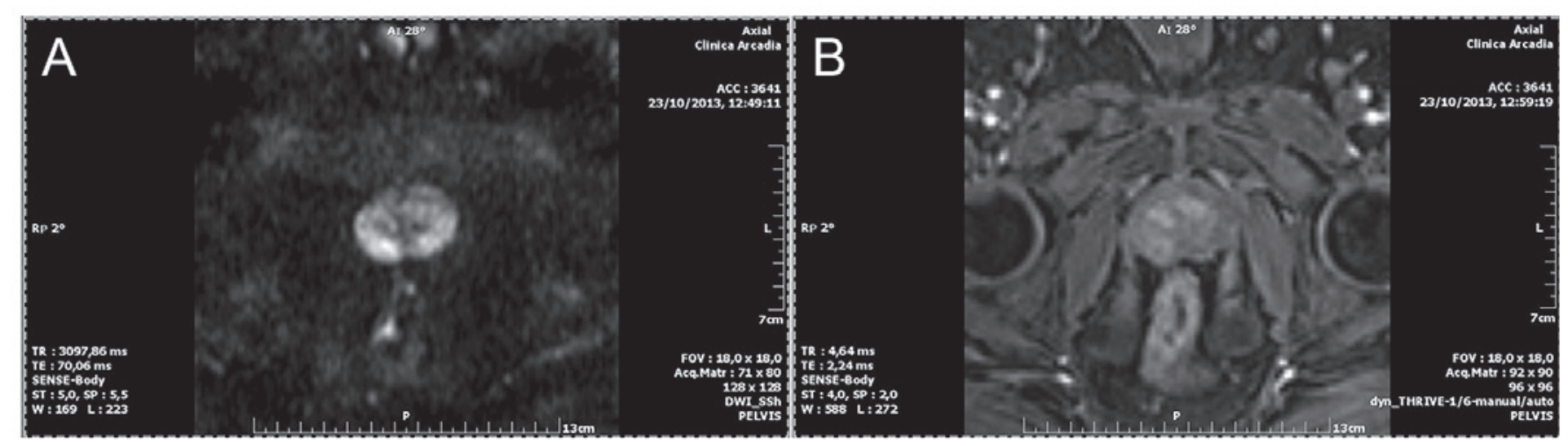

Figure 2. (A) Restricted diffusion area in the peripheral region of both prostate lobes. (B) Contrast enhancement area in both prostate lobes.

(3 instillations). To evaluate the prostate lesions, we decided to perform a second MRI examination 3 months after the first MRI scan. The MRI findings were a 6.4-mm marginal thickening of the left bladder wall and prostate lesions suggestive of malignancy (low T2 signal intensity, restricted diffusion area and contrast enhancement) in both lobes: an area with a maximum diameter of $23.3 \mathrm{~mm}$ in the right lobe and an area of $14 \mathrm{~mm}$ in the left lobe (Fig. 2A and B). The progressive nature of the prostate lesions (involvement of both lobes) was not associated with an increase in PSA levels, which were marginally decreased $(3.6 \mathrm{ng} / \mathrm{ml})$.

Under those conditions (irregularity of the left bladder wall and progression of the prostate lesions suggesting malignant degeneration), we considered that the cardiac risk of general anesthesia in order to perform a more invasive local procedure was justified. Thus, we performed TURB (although, macroscopically, the bladder mucosa was normal) and transrectal ultrasound (TRUS)-guided biopsy (although the suspicious prostate areas were inhomogeneous rather than hypoechoic) and collected 7 samples from the right lobe and 3 from the left lobe (3). The histopathological examination revealed a granulomatous inflammation with giant epithelioid cells, without malignant cells, in any of the bladder and prostate samples.

As the patient was in a good general condition, without any micturition-related complaints, periodical evaluation with DRE, PSA, TRUS and even MRI and the continuation of BCG immunotherapy were recommended.

\section{Discussion}

Granulomatous prostatitis secondary to BCG immunotherapy is a well-known pathological entity (4); however, due to its low incidence, it tends to be overlooked. Previous studies based on histopathological reports $(5,6)$ proved that $75-100 \%$ of the patients who undergo BCG instillations develop granulomatous inflammatory lesions in the prostate. In the majority of the cases the patients are asymptomatic and only rarely (0.9-1.3\%) are there clinical complaints, slight induration of the prostate or elevated PSA level $(7,8)$. In these patients, imaging examinations (TRUS, CT and MRI) may exhibit a number of similarities to prostate cancer (9), for which reason a prostate biopsy may be mandatory to determine the diagnosis (7). The diagnosis of granulomatous prostatitis does not require specific therapy with antibiotics (izoniazid and rifampicin for 3-6 months) in the absence of clinical symptoms, but rather close monitoring (10), which may be a major issue due to the poor data availability in the literature regarding the progression of local findings and PSA level alterations. Under such conditions, we consider that regular complete evaluation (DRE, PSA, TRUS and even MRI) may help avoid further unnecessary prostate biopsy, particularly in patients at high risk due to associated illness.

This case report was an apparently common case of non-muscle invasive bladder tumor that presented with a series of particularities in the patient (previous heavy smoker, 
low pain tolerance, induction of cardiac arrhythmia with anaesthesia and stress exacerbation) as well as in the MRI findings, suggesting a possible associated prostate carcinoma. Radiologists agree that low T2 signal intensity, diffusion restriction and contrast enhancement on MRI are specific to prostate cancer (particularly in the peripheral areas), although some of these findings, particularly low T2 signal in the transition zone, may be specific to granulomatous prostatitis (9). Specialized literature confirms that BCG prostatitis is indicated by low T2 signal on MRI, but does not present data that BCG inflammatory prostatitis may present with all the characteristics of prostate carcinoma, namely diffusion restriction and contrast enhancement in peripheral lesions. Furthermore, following BCG immunotherapy, it is possible to observe a thickening of the bladder wall on MRI examination (9), which was the case in our patient, at 6 months after TURB. The difficulty of imaging differentiation between granulomatous prostatitis and prostate cancer, the possibility of bladder tumor recurrence and the progressive nature of the prostate lesions indicated by repeated MRI examinations, prompted us to perform invasive bladder/prostate procedures, although all the other examinations (DRE, PSA, TRUS and CT) were not suggestive of prostate malignancy. To improve the accuracy of MRI examination in prostate cancer detection, radiologists recommend an MRI device with a 3-Tesla (3-T) magnet and an endorectal coil that may enable a better multi-parametric examination (high-resolution T2-weighted images, dynamic contrast-enhanced, diffusion-weighted imaging/apparent diffusion coefficient maps, MR spectroscopic imaging) and a more correct assessment of Prostate Imaging Reporting and Data System score (11). It would be optimal, for the patient as well as for the urologist, that prostate malignancy was excluded by histopathological examination; in addition, the identical MRI presentation may lead to difficulties in further monitoring the prostate. If the MRI image of the prostate remains unchanged, prostate monitoring should be performed by DRE, PSA measurement and TRUS, so that repeated prostate biopsy (saturation protocol) is the last diagnostic option, performed only if new elements, clearly suggestive of prostate cancer, appear.

In conclusion, we must consider that there are several clinical, biological and imaging similarities between granulomatous prostatitis and prostate cancer. In patients with normal
DRE and normal PSA levels who recently underwent BCG immunotherapy, an abnormal MRI imaging may not be considered strongly indicative of prostate cancer, even if the imaging characteristics are specific to cancer. A multi-parametric MRI examination, using a 3-T magnet and an endorectal coil, may be more useful in differentiating between prostate pathologies. The decision to perform a prostate biopsy must be well justified, to avoid unnecessary procedures, particularly in patients with a high procedure risk due to associated illness.

\section{References}

1. Babjuk M, Burger M, Zigeuner R, et al; European Association of Urology: EAU guidelines of non-muscle-invasive urothelial carcinoma of the bladder: update 2013. Eur Urol 64: 639-653, 2013.

2. Lamm DL, Blumenstein BA, Crissman JD, et al: Maintenance bacillus Calmette-Guerin immunotherapy for recurrent Ta, T1 and carcinoma in situ transitional cell carcinoma of the bladder: a randomized Southwest Oncology Group Study. J Urol 163: 1124-1129, 2000.

3. Heidenreich A, Bellmunt J, Bolla M, et al; European Association of Urology: EAU guidelines on prostate cancer. Part 1: screening, diagnosis, and treatment of clinically localised disease. Eur Urol 59: 61-71, 2011

4. Lamm DL, Stogdill VD, Stogdill BJ and Crispen RG: Complications of bacillus Calmette-Guérin immunotherapy in 1,278 patients with bladder cancer. J Urol 135: 272-274, 1986.

5. LaFontaine PD, Middelman BR, Graham SD Jr and Sanders WH: Incidence of granulomatous prostatitis and acid-fast bacilli after intravesical BCG therapy. Urology 49: 363-366, 1997.

6. Oates RD, Stilmant MM, Freedlund MC and Siroky MB: Granulomatous prostatitis following bacillus Calmette-Guérin immunotherapy of bladder cancer. J Urol 140: 751-754, 1988.

7. Lamm DL, van der Meijden PM, Morales A, et al: Incidence and treatment of complication of bacillus Calmette-Guérin intravesical therapy in superficial bladder cancer. J Urol 147: 596-600, 1992.

8. Leibovici D, Zisman A, Chen-Levyi Z, et al: Elevated prostate specific antigen serum levels after intravesical instillation of bacillus Calmette-Guérin. J Urol 164: 1546-1549, 2000.

9. Ma W, Kang SK, Hricak H, Gerst SR and Zhang J: Imaging appearance of granulomatous disease after intravesical Bacille Calmette-Guérin (BCG) treatment of bladder carcinoma. AJR Am J Roentgenol 192: 1494-1500, 2009.

10. Witjes JA, Palou J, Soloway M, et al: Clinical practice recommendations for the prevention and management of intravesical therapy-associated adverse events. Eur Urol (Suppl 7): 667-674, 2008.

11. Barentsz JO, Richenberg J, Clements R, et al; European Society of Urogenital Radiology: ESUR prostate MR guidelines 2012. Eur Radiol 22: 746-757, 2012. 